\title{
Segmentation of Crack and Open Joint in Sewer Pipelines Based on CCTV Inspection Images
}

\author{
Tung-Ching $\mathrm{Su}$ \\ Department of Civil Engineering and Engineering Management, \\ National Quemoy University, \\ Kinmen, 89250, Taiwan
}

\begin{abstract}
Sewerage, one of major underground pipelines, is an important infrastructure for a modern city. In order to keep sewerage in a good structure and performance condition, planned routine inspection and rehabilitation are necessary. At present, image processing and artificial intelligence techniques have been used to develop diagnostic systems to assist engineers in interpreting sewer pipe defects on CCTV images to overcome human's fatigue and subjectivity, and time-consumption. Based on the segmented morphologies on images, the diagnostic systems were proposed to diagnose sewer pipe defects. This paper proposes a novel method of computer vision, morphological segmentation based on edge detection (MSED), to segment defects in sewer pipelines. In addition to MSED, the traditional image segmentation methods, including opening top-hat operation (OTHO) and closing bottom-hat operation (CBHO), were also applied to the defect segmentation. The historical inspection data revealed that crack and open joint were the two typical sewer pipeline defects in Taiwan, and the experimental result demonstrates that MSED and OTHO are useful for the segmentation of crack and open joint, respectively.
\end{abstract}

Keywords-sewer pipeline; crack; open joint; image segmentation; edge detection; opening top-hat; closing bottom-hat.

\section{INTRODUCTION}

Up-to-date the covering percentage of sewer systems is reported as $35.29 \%$ in Taiwan[1], so the pipeline authorities have been making efforts to construct sewer systems in order to increase the current covering percentage. Once the sewer systems are operated, the issue of sewer inspection and rehabilitation will be faced in the future. At present, closed circuit television (CCTV) is the most popular equipment for sewer inspection because of its low cost compared with sewer scanner evaluation technology (SSET) cameras, ground piercing radar (GPR), sonar, and infrared thermograph[2]. However, human's fatigue and subjectivity, and time-consumption engineers may be barriers for detecting and diagnosing defects in sewer pipelines via CCTV images due to the great number of inspected images. Recently, image processing and artificial intelligence techniques were applied as diagnostic systems to assist engineers in interpreting sewer pipe defects on inspection images[3].

Mathematical morphology-based image segmentation, one of the image processing techniques, has been extensively applied to the researches of pattern recognition[4]. Erosion and dilation are two basic operators of morphological segmentation, and are usually operated in tandem for the image enhancement of interested objects[5]. Opening top-hat operation (OTHO), in which firstly erosion followed by dilation (called image opening) was applied to an SSET inspection image, secondly the opening operated image was subtracted from its SSET inspection image (called top-hat operation), and finally the top-hat operated image was transferred into a binary one by Otsu's technique, was used to segment pipeline defects, including crack, hole/joint, laterals, and pipe collapse, in sewer pipelines[4]. However, environmental noise or poor image quality would deteriorate the performance of OTHO so to hamper the pipeline defect detection.

Due to the dark environment inside sewerage, a beam provided by CCTV robot is needed during sewer inspection. The optic axis of CCTV robot and the center axis of pipeline are usually parallel. Consequently, open joint is apt to be illuminated by CCTV robot because the surface of open joint is usually perpendicular to the center axis of pipeline. Cracks in CCTV images could be regarded as clearly dark patterns[6], but sometimes appear bright patterns under several specific imaging conditions. Closing bottom-hat operation (CBHO), which is the dual operation of OTHO and useful in dark pattern segmentation, was also taken into consideration of detecting the pipeline defects in this research. In addition to OTHO and $\mathrm{CBHO}$, this paper presents a novel algorithm, morphological segmentation based on edge detection (MSED), in order to effectively and correctly segment sewer pipeline defects from CCTV inspection images.

\section{Methodology}

Figure 1 shows the schematic outline of this research. The involved techniques are introduced as follows. 


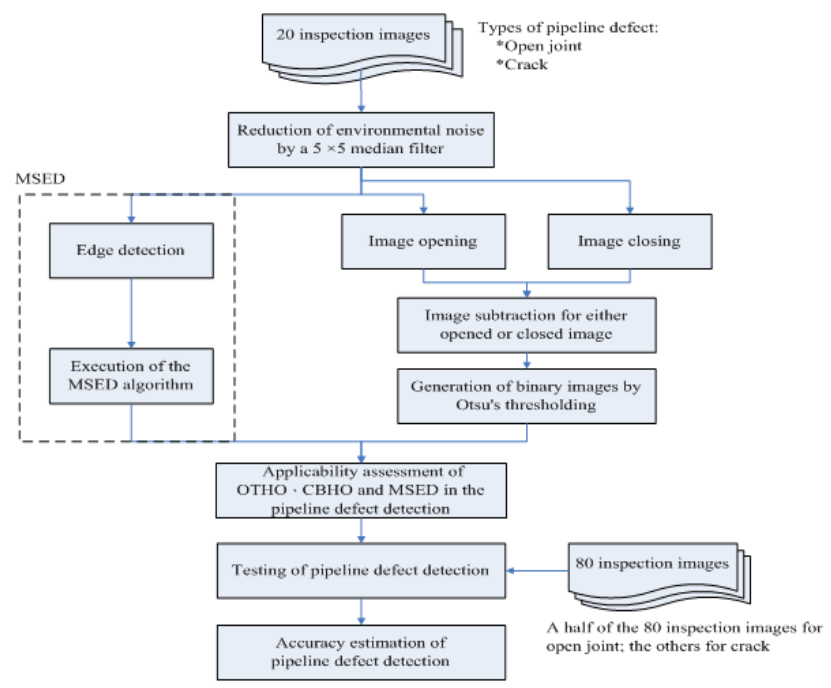

Figure 1. Research scheme

\section{A. Median filtering}

Due to the discreteness of digital numbers, an inspection image I can be expressed as

$$
\mathrm{I}=\left(I_{1}, I_{2}, \ldots, I_{N}\right)
$$

where $N$ is the number of total pixels in $\mathbf{I}$. The size and shape of a kernel must be determined prior to median filtering. A square kernel consisting of $M \times M$ elements is usually applied to median filtering[7]. Generally, $M$ must be an odd number above or equal to $3(M=5$ adopted in this paper) so that kernel has the only central element to be filtered.

\section{B. Opening top-hat operation (OTHO)}

Sets $\mathbf{M}$ and $\mathbf{S}$ represent a median filtered image consisting of pixels $p(x, y)$ and a structuring element, respectively.

$$
\begin{gathered}
\mathbf{M}=\{(x, y) \mid p(x, y)\} \\
\mathbf{S}=\{(x, y) \mid(x, y) \text { in structuring element }\} .
\end{gathered}
$$

The dilation of $\mathbf{M}$ by $\mathbf{S}$, denoted $\mathbf{M} \oplus \mathbf{S}$, is the union of all pixels in $\mathbf{M}$ surrounded by the shape of $\mathbf{S}$ and defined as

$$
\mathbf{M} \oplus \mathbf{S}=\{m+s \mid \text { for all } m \in \mathbf{M} \text { and } s \in \mathbf{S}\} .
$$

Similarly, the erosion of $\mathbf{M}$ by $\mathbf{S}$, denoted $\mathbf{M} \Theta \mathbf{S}$, removes all pixels within a "distance" $\mathbf{S}$ from the edge of $\mathbf{M}$ and is defined as

$$
\mathbf{M} \Theta \mathbf{S}=\{m \mid s+m \in \mathbf{M} \text { for every } s \in \mathbf{S}\} .
$$

Based on Eqs. (4) and (5), the opening operation is defined as

$$
\text { M。 } \mathbf{S}=(\mathbf{M} \Theta \mathbf{S}) \oplus \mathbf{S} \text {. }
$$

Image regions rarely relative to the structuring element are removed by the opening operation while preserving image regions greater than structuring elements[4]. Based on Eq. (6), OTHO is defined as

$$
\mathrm{OTHO}_{\mathbf{M} \circ \mathbf{S}}=\mathbf{M}-(\mathbf{M} \Theta \mathbf{S}) \oplus \mathbf{S} .
$$

In inspection image, open joint looks like the object of illuminated ring, but crack usually presents in an irregular or linear pattern. There is a great difference between the patterns of open joint and crack, so a disk SE due to its all-round shape was adopted to assist OTHO in morphologically probing the pipeline defects. After implementing OTHO, a binary image, in which the ' 1 ' pixels express interested objects and the ' 0 ' pixels denote background environment, is produced.

\section{Closing bottom-hat operation (CBHO)}

CBHO is the dual operation of OTHO. Also, a disk SE was introduced into $\mathrm{CBHO}$, and a binary image is produced after implementing $\mathrm{CBHO}$. Image dilation and erosion are the two basic operations of image opening and closing, but image closing is just the inverted operation of image opening and defined as

$$
\mathbf{M} . \mathbf{S}=(\mathbf{M} \oplus \mathbf{S}) \Theta \mathbf{S} .
$$

Based on Eq. (8), CBHO is defined as

$$
\mathrm{CBHO}_{\mathrm{M} \cdot \mathrm{S}}=(\mathbf{M} \oplus \mathbf{S}) \Theta \mathbf{S}-\mathbf{M} \text {. }
$$

\section{Morphological segmentation based on edge detection (MSED)}

Based on an edge detection result, the MSED algorithm attempts to transform the black pixels between two detected edges into white pixels. In this research, the Sobel edge detector of first derivative was taken into consideration of the edge detection of the pipeline defects in the median filtered image $\mathbf{M}$. The MSED algorithm applies region growing to the morphological segmentation of pipeline defect based on the edge detection result, and was encoded in Matlab. In the following algorithm, $d_{\text {lim }}$ is used to survey the minimum distance between two border pixels in either row or column direction. Once the minimum distance is determined, the interval pixels, i.e. the ' 0 ' pixels, are changed into the interested pixels, i.e. the ' 1 ' pixels.

The MSED algorithm:

$\mathrm{d}=2: d_{\text {lim }} \% d_{\text {lim }}$ is an integer; loops $\mathrm{d}$ from 2 to $d_{\text {lim }}$

If (image $(i, j)==1 \&$ image $(i, j+d)==1) \%$ image $(i$,

$\mathrm{j})$ : A binary image produced by edge detection

image $(i, j+1: j+d-1)=1 ; \%$ region growing in the column direction

End

If (image $(i, j)==1 \&$ image $(i+d, j)==1)$

image $(i+1: i+d-1, j)=1 ; \%$ region growing in the row direction

End 


\section{E. Accuracy estimation of pipeline defect segmentation}

To assess the applicability of the three methods in pipeline defect segmentation, the accuracy indices, including completeness (Compl), correctness (Corr), and quality, are introduced as:

$$
\begin{gathered}
\text { Compl }=\left(S_{e} \cap M_{s}\right) / M_{s} \\
\text { Corr }=\left(S_{e} \cap M_{s}\right) / S_{e} \\
\text { Quality }=(\text { Compl } \times \text { Corr }) /(\text { Compl }- \text { Compl } \times \text { Corr }+ \text { Corr }) .
\end{gathered}
$$

where $S_{e}$ is the segmentation by OTHO, CBHO or MSED method; $M_{S}$ expresses the image regions of true pipeline defects interpreted by experts.

\section{RESULT AND DISCUSSION}

This paper acquired 100 frames of inspection images as experimental materials, and 20 of the 100 inspection images were selected as the training samples to see the performance of the three segmentation methods in the pipeline defect segmentation. Crack and open joint both were given 10 training sample images, which were numbered as (a) through (j). Fig. 2 presents two sample images for crack and open joint.

\section{A. Performance of OTHO, CBHO and MSED in the pipeline defect segmentation}

Table I lists the accuracies of crack and open joint segmentations, respectively, and show OTHO performing better in open joint segmentation than crack segmentation. For the crack segmentation, the best Compl of $71.60 \%$ was obtained because the crack in Image (j) was strong illuminated. However, the transverse stripe of non-crack and the inspection texts in Image (j) were also segmented so that the obtained Corr is merely 8.64\%. Table 1 illustrates that OTHO is really unsuitable for crack segmentation. In addition, OTHO offering Compl above $85 \%$ for open joint segmentation indicates that most image regions belonging to open joints can be segmented. However, not only the open joints but also the inspection texts or the noisy environments were segmented so that the Corr is far inferior to the Compl.

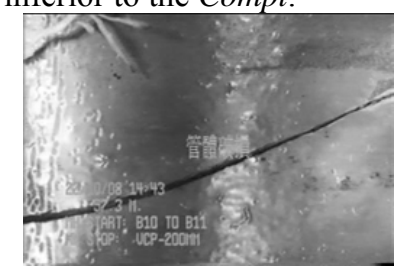

(a) crack (b) open joint

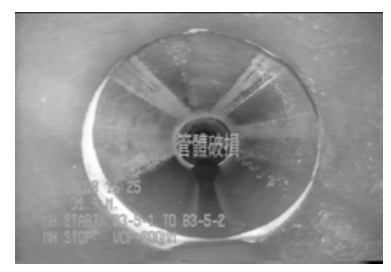

Figure 2. Sample images

The accuracy of the segmented pipeline defects by $\mathrm{CBHO}$ is estimated as Table II. Obviously, CBHO derives the better performance in segmenting crack than open joint that is just contrary to the effect of OTHO. Compared with
Table I and II, the Quality of crack segmentation is significantly improved. Such result demonstrates that $\mathrm{CBHO}$ is suitable to detect object which absorbs beam. Most of the segmented cracks can be given Compl above $50 \%$ by CBHO. In particular, the crack in Image (e) obtained the Compl of $82.79 \%$. Also, the inspection text or the noisy environment was detected by $\mathrm{CBHO}$ so that the crack in Image (e) has the Corr of $8.94 \%$ which is far inferior to the Compl of $82.79 \%$. Consequently, the low Corr results in the low Quality of $8.78 \%$. Table II also shows that $\mathrm{CBHO}$ is unsuitable for open joint segmentation due to the values of Quality mostly approximating to $0 \%$. Even, Image (e) was given the Quality of $0 \%$ with Compl and Corr both being $0 \%$.

In Table III, MSED offers the better performance for open joint segmentation than crack segmentation. A comparison between Tables II and III shows MSED superior to $\mathrm{CBHO}$ in crack segmentation due to the less noisy environment segmentation by MSED. In Table III, the best Quality of $26.51 \%$ with Compl of $80.29 \%$ and Corr of $28.36 \%$ was obtained for the crack segmentation in Image (c). Especially, the Corr of $28.36 \%$ can be greatly improved if the inspection texts were not overlaid onto the image. Similarly, in Table III the lower Corrs, such as $7.60 \%, 5.11 \%$ or $5.12 \%$, resulted from the significant segmentation of the inspection texts so to deteriorate the Quality of $6.91 \%, 5.01 \%$ or $5.03 \%$. For open joint segmentation, the four segmented images, including Image (d), (e), (f), and (g), obtained better Quality. Especially for Image (e), the Quality of $54.47 \%$ with Compl of $90.89 \%$ and Corr of $57.62 \%$ was the optimum derived by MSED.

\section{B. Verification of OTHO and MSED in pipeline defect segmentation}

The above result demonstrates that OTHO and MSED can be considered as suitable for open joint and crack segmentations, respectively. Based on the other 80 images, Figure 3 shows the obtained Compl, Corr, and Quality for the applicability verification. In Figure 3, the bars represent the accuracies of MSED and OTHO in the crack and open joint segmentations, respectively. The longer bar resulted in greater difference between the maximum and minimum of the obtained accuracies. Figure 3 shows the averaged Compl of $86.72 \%$ (standard deviation= $13.59 \%$ ) and $61.50 \%$ (standard deviation $=19.98 \%$ ) for the open joint and crack segmentations, respectively. This result indicates that OTHO has a robust capability of segmenting open joints and MSED can effectively segment cracks. The Corr of the open joint segmentation ranges significantly wider than that of the crack segmentation. The standard deviations of Corr for the open joint and crack segmentations are $18.49 \%$ and $5.89 \%$, respectively. The statistic result of the Corr is similar to that of the Quality. Figure 3 also shows that the Corr is greatly inferior to the Compl due to the influence of the noisy environment. Moreover, it would be more difficult to correctly segmenting crack than open joint so that the Quality of crack segmentation is relatively lower than that of open joint segmentation. 
TABLE I. ACCURACY OF CRACK (C) AND OPEN JOINT (OJ) SEGMENTATIONS BY OTHO

\begin{tabular}{crrrrrr}
\hline \multirow{2}{*}{ Image ID } & \multicolumn{2}{c}{ Compl } & \multicolumn{3}{c}{ Accuracy index (\%) } \\
& C & OJ & C & OJ & C & Ou J \\
\hline (a) & 8.28 & 98.26 & 2.80 & 49.25 & 2.14 & 48.82 \\
(b) & 6.91 & 88.14 & 3.39 & 41.12 & 2.33 & 38.97 \\
(c) & 2.44 & 83.92 & 1.60 & 31.32 & 0.97 & 29.55 \\
(d) & 1.56 & 91.73 & 0.28 & 47.33 & 0.23 & 45.40 \\
(e) & 1.41 & 88.16 & 0.19 & 74.39 & 0.17 & 67.64 \\
(f) & 19.93 & 86.21 & 4.77 & 55.92 & 4.00 & 51.33 \\
(g) & 5.40 & 87.43 & 0.91 & 56.24 & 0.79 & 52.03 \\
(h) & 0.56 & 92.66 & 0.07 & 19.21 & 0.07 & 18.93 \\
(i) & 9.57 & 92.56 & 0.78 & 39.66 & 0.73 & 38.44 \\
(j) & 71.60 & 91.30 & 8.64 & 47.97 & 8.35 & 45.88 \\
\hline
\end{tabular}

TABLE II. ACCURACY OF CRACK (C) AND OPEN JOINT (OJ) SEGMENTATIONS BY CBHO

\begin{tabular}{crrrrrr}
\hline & \multicolumn{9}{c}{ Accuracy index (\%) } \\
Image ID & \multicolumn{2}{c}{ Compl } & \multicolumn{2}{c}{ Corr } & \multicolumn{2}{c}{ Quality } \\
& \multicolumn{1}{c}{ C } & OJ & \multicolumn{1}{c}{ C } & OJ & C & OJ \\
\hline (a) & 27.48 & 6.89 & 9.56 & 3.14 & 7.63 & 2.21 \\
(b) & 56.89 & 0.51 & 21.89 & 0.43 & 18.77 & 0.23 \\
(c) & 71.39 & 0.12 & 39.35 & 0.18 & 33.99 & 0.07 \\
(d) & 70.18 & 0.11 & 11.51 & 0.03 & 10.97 & 0.03 \\
(e) & 82.79 & 0.00 & 8.94 & 0.00 & 8.78 & 0.00 \\
(f) & 53.43 & 2.65 & 22.21 & 4.58 & 18.61 & 1.71 \\
(g) & 52.16 & 2.35 & 6.21 & 4.46 & 5.87 & 1.57 \\
(h) & 65.86 & 2.25 & 9.75 & 2.30 & 9.28 & 1.15 \\
(i) & 79.65 & 10.66 & 11.72 & 2.45 & 11.37 & 2.03 \\
(j) & 4.94 & 1.16 & 0.73 & 0.36 & 0.64 & 0.28 \\
\hline
\end{tabular}

TABLE III. ACCURACY OF CRACK (C) AND OPEN JOINT (OJ) SEGMENTATIONS BY MSED

\begin{tabular}{crrrrrr}
\hline & \multicolumn{9}{c}{ Accuracy index (\%) } \\
Image ID & \multicolumn{2}{c}{ Compl } & \multicolumn{2}{c}{ Corr } & \multicolumn{2}{c}{ Quality } \\
& C & OJ & \multicolumn{1}{c}{ C } & OJ & C & OJ \\
\hline (a) & 43.21 & 50.17 & 7.60 & 24.70 & 6.91 & 19.84 \\
(b) & 49.51 & 64.02 & 25.47 & 30.96 & 20.22 & 26.37 \\
(c) & 80.29 & 58.22 & 28.36 & 22.44 & 26.51 & 19.33 \\
(d) & 67.45 & 97.70 & 12.57 & 37.31 & 11.85 & 36.98 \\
(e) & 76.53 & 90.89 & 12.23 & 57.62 & 11.79 & 54.47 \\
(f) & 62.14 & 87.38 & 22.59 & 38.87 & 19.86 & 36.80 \\
(g) & 72.30 & 88.87 & 5.11 & 37.47 & 5.01 & 35.79 \\
(h) & 83.30 & 71.50 & 17.87 & 15.58 & 17.25 & 14.67 \\
(i) & 58.20 & 20.03 & 18.17 & 10.63 & 16.07 & 7.46 \\
(j) & 73.66 & 62.77 & 5.12 & 33.74 & 5.03 & 28.11 \\
& & & & & & \\
\hline
\end{tabular}

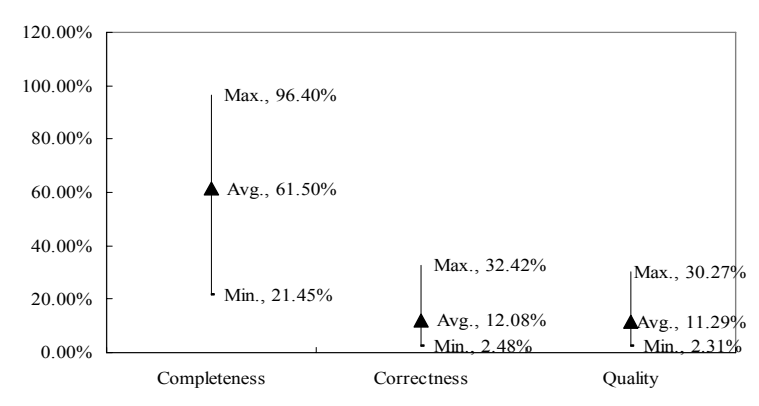

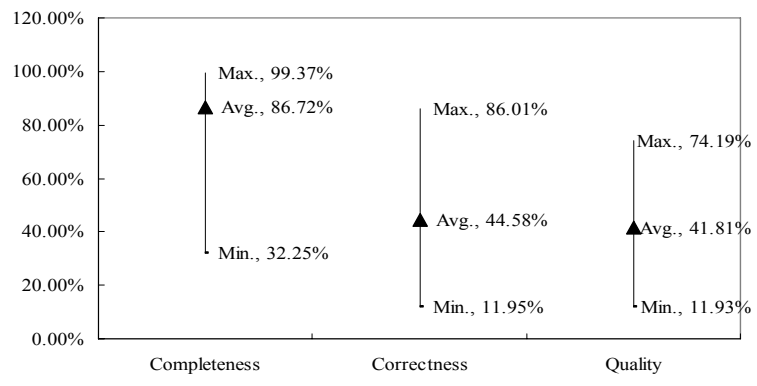

$\begin{array}{ll}\text { (a) crack segmentation } & \text { (b) open joint segmentation }\end{array}$

Figure 3. MSED and OTHO in crack and open joint segmentations, respectively

\section{CONCLUSIONS}

This paper demonstrates that OTHO and MSED have better performance in open joint and crack segmentations, respectively. Crack was found more difficult for segmentation due to its various types of appearance. MSED compared with $\mathrm{CBHO}$ can effectively improve the quality for crack segmentation. However, the inspection texts recorded onto the acquired CCTV images deteriorate the correctness and quality. We strongly suggest that a separate attribute database for inspection texts is necessary in the future CCTV shooting process. Removal of the inspection texts from CCTV images should significantly improve the detection correctness. In the further work, LiDAR will be introduced into pipeline defect measurement based on point cloud with precise coordinates.

\section{REFERENCES}

[1] Construction and Planning Agency, Ministry of the Interior Website. Available online: http://www.cpami.gov.tw/chinese/index.php?option=com_content\&v iew $=$ article $\& i d=9995 \&$ Itemid $=53$ (accessed on 4 March 2014).

[2] Makar, J.M., Diagnostic techniques for sewer systems. Journal of Infrastructure Systems, 5(2), pp.69-78,1999.

[3] Yang, M.D. \& T.C. Su. Segmenting ideal morphologies of sewer pipe defects on CCTV images for automated diagnosis. Expert Systems with Applications, 36(2), pp.3562-3573,2009.

[4] Sinha, S.K. \& P.W. Fieguth. Segmentation of buried concrete pipe images. Automation in Construction, 15(1), pp.47-57,2006.

[5] Hsiao, Y.T., Chuang, C.L., Lu, Y.L. \& J.A. Jiang. Robust multiple objects tracking using image segmentation and trajectory estimation scheme in video frames. Image and Vision Computing, 24(10), pp.1123-1136,2006.

[6] Yang, M.D. \& T.C. Su. Automated diagnosis of sewer pipe defects based on machine learning approaches. Expert Systems with Applications, 35(3), pp.1327-1337,2008.

[7] Morillas, S., Gregori, V. \& A. Sapena. Adaptive marginal median filter for colour images. Sensors, 11(3), pp.3205-3213,2011. 